\title{
Search for WIMPs with background evaluation in a fiducial volume by XMASS-I
}

\author{
Hiroshi Ogawa for the XMASS collaboration* \\ Kamioka Observatory, Institute for Cosmic Ray Research, the University of Tokyo \\ Higashi-Mozumi, Kamioka, Hida, Gifu, 506-1206, Japan \\ Kavli Institute for Physics and Mathematics for the Universe, the University of Tokyo \\ Kashiwa, Chiba 277-8582, Japan \\ E-mail: ogawa@suketto.icrr.u-tokyo.ac.jp
}

\begin{abstract}
XMASS is a large volume multi-purpose experiment using a liquid xenon technology located underground in Kamioka Observatory, Japan. XMASS-I aims to search for dark matter, which is holding $832 \mathrm{~kg}$ of liquid xenon in an active volume. In this talk, the result of WIMPs search using fiducial volume analysis will be reported. The data accumulated between November 2013 and March 2016 were used for this analysis with 705.9days livetime. We present the WIMPs search with precise prediction of background event.
\end{abstract}

35th International Cosmic Ray Conference - ICRC2017

10-20 July, 2017

Bexco, Busan, Korea

* Speaker.

${ }^{\dagger}$ A footnote may follow. 


\section{Introduction}

XMASS is a multi-purpose detector using ultra pure liquid xenon. Its main physics target is direct dark matter (DM) detection.

The XMASS-I detector [1] was constructed at the Kamioka Laboratory with an overburden of with 2700 m.w.e. A cylindrical water tank of $10 \mathrm{~m}$ diameter and $11 \mathrm{~m}$ height contains 72 20-inch PMTs and ultra pure water. It serves as a shield against fast neutrons and external gammas as well as an active muon veto. $832 \mathrm{~kg}$ LXe is filled as active scintillation target. 642 PMTs are mounted on the inner surface of the detector about $40 \mathrm{~cm}$ radius.

XMASS-I experiment had been started from Dec.2010. Physics data taking is continuing except detector refurbishment term from Aug.2012 to Oct.2013.

XMASS-I is a single phase detector. The simplicity of its design allows for easy scaling to larger detector sizes its high photo-coverage for large light yield which achieves low threshold about $0.3 \mathrm{keVee}$. LXe has large atomic number $(\mathrm{Z}=54)$ and high density $(\sim 3 \mathrm{~g} / \mathrm{cc})$. The effective external background reduction ('self shielding') are enough by vertex reconstruction. Dark matter search for XMASS-I is used these advantage effectively. Low threshold achieved the low mass WIMPs search [2] and annual modulation analysis [3]. Fiducial volume analysis using vertex reconstruction achieved the high sensitive search for Bosonic super-WIMPs [4] and DM Xe129 inelastic mode search [5].

In this paper, WIMPs are searched with the background subtraction in the fiducial volume for XMASS-I. For this purpose, the background evaluation is summarized in Section 2, which also discusses the Geant 4 Monte Carlo simulation and radioactivity from detector material . In section 3 , the reduction of data is explained for each reduction steps. Also the remaining events from their radioactive contamination in the detector developed and the liquid xenon are explained with systematic error evaluation. The WIMPs search is presented with comparison of background MC and data in Section 4.

\section{Background evaluation in XMASS-I}

Analysis in this paper requires precise evaluation of expected background event in XMASS-I. For this purpose, Geant4 based XMASS Monte Carlo (XMASS MC) simulation was constructed. The detector geometry, materials and its radioactivities are reproduced. XMASS MC is also fully reproduced the generation of scintillation photons and PMT response.

RI generation in XMASS MC is varified against HPGe screening results obtained for our detector materials, except for copper and LXe. Activity in XMASS is also derived from spectrum fitting background MC with variable component activity to 15 days of real data without fiducial volume cut. Fitting includes only events with more than about 400pe. The systematic error in the inferred activity (activity error) is estimated by varying the individual contributions within their respective uncertainties (energy scale, detector response and so on). Figure 1 shows the energy spectrum for each RI component and error with real data. Difference of spectrum shape is within activity error except between 20000 and 25000pe.

The RI activities in the copper and LXe, which are small contribution to the spectrum without a fiducial volume cut, are estimated independently. The copper material is used to detector surface, 
detector holder and so on. Copper includes some amount of ${ }^{210} \mathrm{~Pb}$. The alpha event spectrum in XMASS dataset is studied for activity estimation. Internal RI in the LXe is expected to the from ${ }^{222} \mathrm{Rn},{ }^{85} \mathrm{Kr},{ }^{39} \mathrm{Ar}$ and ${ }^{14} \mathrm{C}$ by analysis of real data. Other background contributions are very small, including those from $(\alpha, \mathrm{n})$ reactions in the detector material, cosmogenic RI, ${ }^{220} \mathrm{Rn}$ and $2 v \beta \beta$ in LXe.

In less than about 400pe, dominant background event is from PMT aluminum seal using PMT surface. ${ }^{238} \mathrm{U}$ and ${ }^{210} \mathrm{~Pb}$ in the aluminum made huge background event in first phase of XMASS-I. Detector refurbishment was processed that PMT aluminum seal was covered by copper ring and plate. Background event is reduced 1/10 after detector refurbishment. Furthermore, small amount of scintillation light leakage is remained from crack of fusion between aluminum seal and quartz. These scintillation light make the amount of low energy event. The shape of NPE distribution depends to the model of aluminum seal shape, crack and aluminum vapor. But the contribution of light leakage from aluminum seal very small after fiducial volume cut. PMT hit pattern of light leakage event is unique and our reconstruction is easy to reject these events.

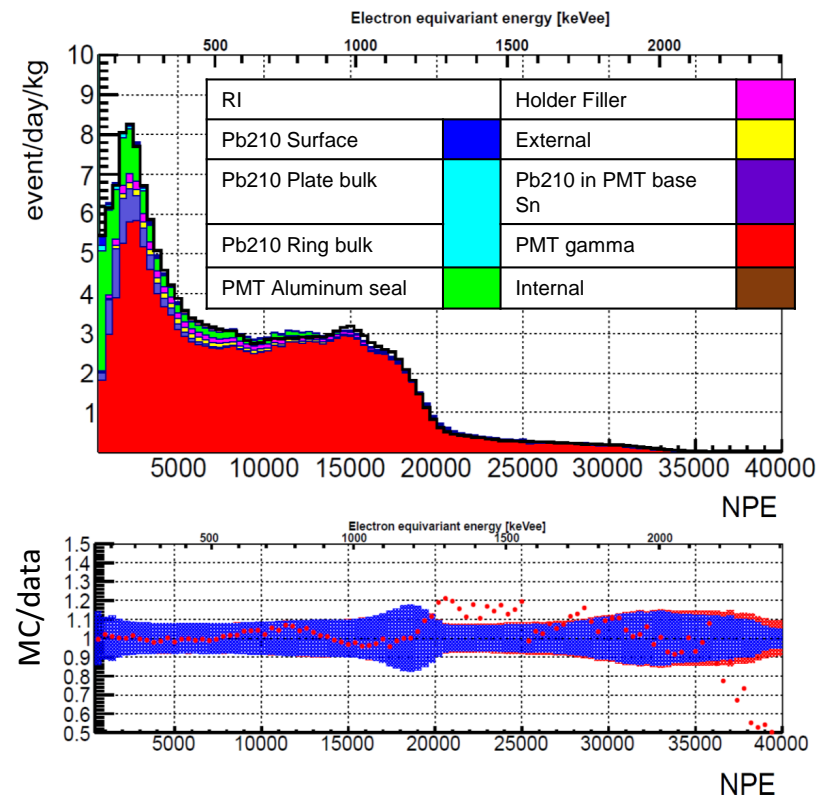

Figure 1: (Preliminary) (top) NPE distribution of spectra for XMASS data and background MC. Black line is data. (bottom) discrepancy between data and background MC. red point is ratio between data and MC. Blue region indicates the uncertainly of radioactivity. Red region indicates the statistics error of Background MC.

\section{Event reduction, background evaluation in fiducial volume with systematic error evaluation}

Event reduction has two steps. First step is named as 'standard cut". 'standard cut' reduces the events that introduced by electronics noise, very short time correlated and Cherenkov like event. Cherenkov like event is mainly generated by $40 \mathrm{~K}$ into PMT photo-cathode and removed by which (number of hit within 20ns) / total number of hit is more than 0.6 and total NPE is less than 200pe. 
After standard cut, the vertex position is reconstructed for fiducial volume cut. Vertex reconstruction method has two type. One is timing based vertex [6] ' $R(T)^{\prime}$ and the other is NPE based vertex [1] $R(P E)^{\prime}$. Reconstructed radius is selected within $38 \mathrm{~cm}$ for $R(T)$ and within $20 \mathrm{~cm}$ for $\mathrm{R}(\mathrm{PE})$ as fiducial volume to reduce the event that is generated in detector surface.

These reduction is applied both real data and background MC commonly. The data accumulated between November 2013 and March 2016 is used for this analysis. After removing periods of operation with excessive PMT noise or data acquisition problems, the total live time of dataset become 705.9days. Background MC is generated using XMASS MC for each RI and its activity. Same event selection are applied to background MC which has same statistics with dataset and traced optical parameter from the estimation of Co57 periodic calibration.

Figure 2 shows the energy distribution applying standard cut, $\mathrm{R}(\mathrm{T})$ and $\mathrm{R}(\mathrm{PE})$ cut for real data and background MC. Left of Figure 2 is separated by component of background RI. The remained background origin in analysis are mainly detector ${ }^{210} \mathrm{~Pb}$ in copper plate end ring which is happened in surface of detector. These events are happened in no sensitive angle of PMT and make mimic as fiducial volume event. we named these event as 'miss-reconstructed event". Also missreconstruction event are made from PMT gamma and PMT aluminum origin. Only LXe internal background events are actually happened in LXe, which are same with DM signal.

Systematic error for fiducial volume analysis were evaluated. Table 1 shows the list of systematic error and its relative value. The condition of detector surface which is corresponding from (1) to (4) in Table1 1 make the large value of systematic error, mainly R(PE) cut. Also uncertainly of reconstruction performance, timing response, absorption and scattering length of LXe including time variation, response near of dead tube and nuclear recoil event in background $\mathrm{MC}$ are included to systematic error. The right of figure 2 shows energy spectrum with total systematic error with real data applying general cut, $\mathrm{R}(\mathrm{T})$ and $\mathrm{R}(\mathrm{PE})$ cut.
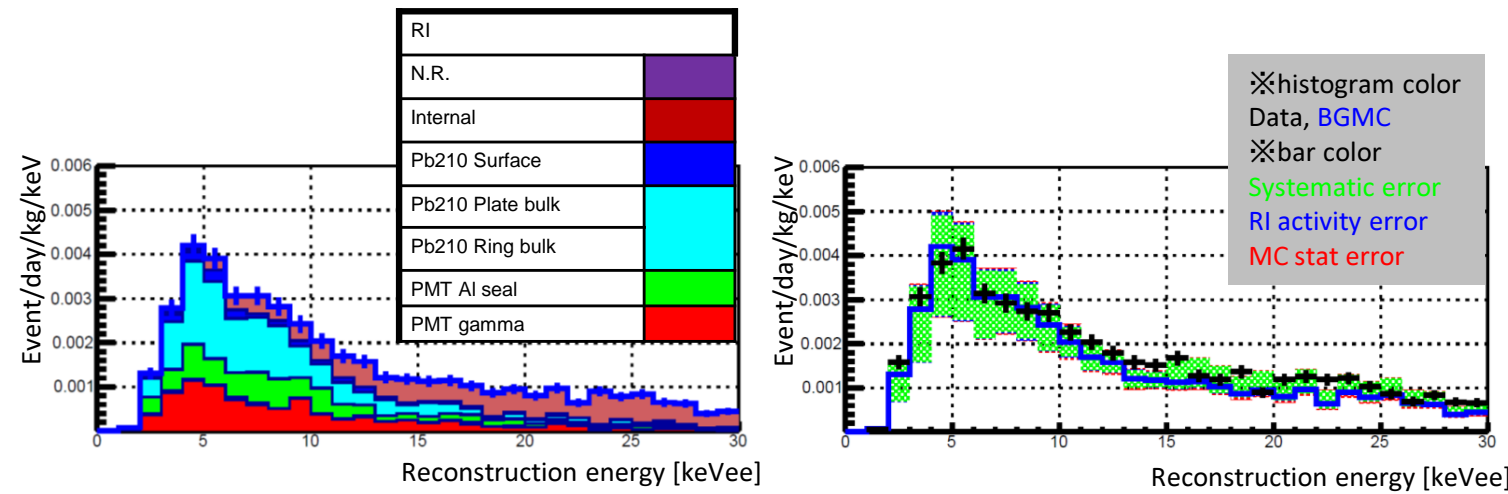

Figure 2: (Preliminary)Left : energy spectrum for each RI for BGMC. Right : energy spectrum of systematic error for BGMC with real data. Both apply general cut and $\mathrm{R}(\mathrm{PE})+\mathrm{R}(\mathrm{T})$ cut.

\section{Dark matter search with fiducial volume cut with background evaluation}

We performed WIMP dark matter search in events of final reduction sample of real data with consideration for background events predicted by background MC. Events induced by WIMP- 
Table 1: (Preliminary)The list of systematic error and its relative value.

\begin{tabular}{|c|c|c|c|}
\hline \multirow[t]{2}{*}{ Contents } & \multicolumn{2}{|c|}{ Systematic error (each event position and RI) } & \multirow[t]{2}{*}{ comment } \\
\hline & 2-15keVee & 15-30keVee & \\
\hline $\begin{array}{l}\text { (1) Plate gap } \\
\text { dependence }\end{array}$ & $+6.2 /-22.8 \%$ & $+1.9 /-6.9 \%$ & Gap 30, 85(current) and 140um \\
\hline (2) Ring roughness & $+6.6 /-7.0 \%$ & $+2.0 /-2.1 \%$ & Impact to beta event \\
\hline (3) Cu ref dependence & $+5.2 /-0.0 \%$ & $+2.5 /-0.0 \%$ & Ref $0.2,0.25$ (current) and 0.3 \\
\hline (4) Plate floating & $+0.0 /-4.6 \%$ & $+0.0 /-1.4 \%$ & The case that $0.6 \mathrm{~mm}$ floating for center of detector. \\
\hline (5) Al seal dependence & $+0.7 /-0.7 \%$ & $+0.0 /-0.0 \%$ & Max : A-kit model, min : no valley contribution \\
\hline (6) Reconstruction & $+3.0 /-6.2 \%$ & $+0.0 /-0.0 \%$ & $\begin{array}{l}\text { Reconstruction grid dependence, thinning sample and } \\
\text { hose run. }\end{array}$ \\
\hline $\begin{array}{l}\text { (7) Timing (decaytime, } \\
\text { TTS) }\end{array}$ & $+4.6 /-2.9 \%$ & $+0.4 /-5.3 \%$ & \multirow{2}{*}{$\begin{array}{l}\text { Impact to Cherenkov distribution. Estimated by } \\
\text { internal source calibration for (7) and external gamma } \\
\text { source calibration for ( } 8 \text { ). }\end{array}$} \\
\hline $\begin{array}{l}\text { (8) Timing (response in } \\
\text { detector surface) }\end{array}$ & $+0.0 /-8.0 \%$ & $+0.0 /-0.0 \%$ & \\
\hline $\begin{array}{l}\text { (9) Absorption \& } \\
\text { scattering }\end{array}$ & $+0.7 /-6.7 \%$ & $+1.5 /-1.1 \%$ & $\begin{array}{l}\text { Mass production : scat length no constraint. } \\
\text { Additional BGMC (Pb210 Plate bulk, ring bulk and } \\
\text { internal) are processed by scat } 52 \text { and } 53 \mathrm{~cm} \text { fixed. } \\
\text { Value in right is scat } 52 \mathrm{~cm} \text { case. }\end{array}$ \\
\hline (10) Dead tube origin & $+10.3 /-0.0 \%$ & $+45.2 /-0.0 \%$ & response of dead tube \\
\hline (11) N.R. & $+0.7 /-0.7 \%$ & $+0.0 /-0.0 \%$ & Q.F. dependence, put on surface or embedded. \\
\hline
\end{tabular}

nucleon elastic scattering were also simulated by XMASS MC (WIMP MC) for each WIMP mass from $10 \mathrm{GeV}$ to $10 \mathrm{TeV}$. We assume a standard spherical isothermal galactic halo model with the most probable speed of $v_{0}=220 \mathrm{~km} / \mathrm{s}$, escape velocity of $v_{\text {esc }}=544 \mathrm{~km} / \mathrm{s}$, and a local dark matter density of $0.3 \mathrm{GeV} / \mathrm{cm}^{3}$, following [7]. Same processes of event reduction were applied to WIMP MC. The systematic error for WIMP MC comes from the uncertainty of absorption and scattering length of LXe including time variation, scintillation decay time, efficiency for event reduction evaluated and scintillation efficiency for nuclear recoils ( $\left.\mathscr{L}_{\text {eff }}\right)$.

Then energy spectrum of real data was fitted with background MC and WIMP MC in the energy range of $2-15 \mathrm{keV}_{\text {ee }}$. Then, the $90 \%$ C.L. upper limit on the WIMP-nucleon cross section was derived in figure 3 Our stringent exclusion limit is $2.2 \times 10^{-44} \mathrm{~cm}^{2}$ for $60 \mathrm{GeV} / \mathrm{c}^{2}$ WIMPs.

Our background $\mathrm{MC}$ revealed that remaining events were mainly caused from miss-reconstruction event. these are happened in no sensitive angle of PMT and make mimic as fiducial volume event and already discussed in section 3. To solve this problem, a new type of the PMT which has a dome-shaped photocathode was developed. The dome-shaped PMT can detect the scintillation photons emitted from the neighbor surface, and reduce miss-reconstructed events drastically.

\section{References}

[1] K. Abe, et al., XMASS collaboration, Nucl. Instr. and Meth. A716 (2013) 78-85.

[2] K.Abe, et al., XMASS collaboration, Phys. Lett. B 719 (2013) 78.

[3] K.Abe, et al., XMASS collaboration, Phys. Lett. B 759 (2016) 272.

[4] K. Abe, et al., XMASS collaboration, Phys Rev Lett, 113, (2014) 121301.

[5] U. Uchida, et al., XMASS collaboration, Prog. Theor. Exp. Phys. (2014) 063C01.

[6] A. Takeda for the XMASS collaboration, 34th International Cosmic Ray Conference (ICRC2015). 


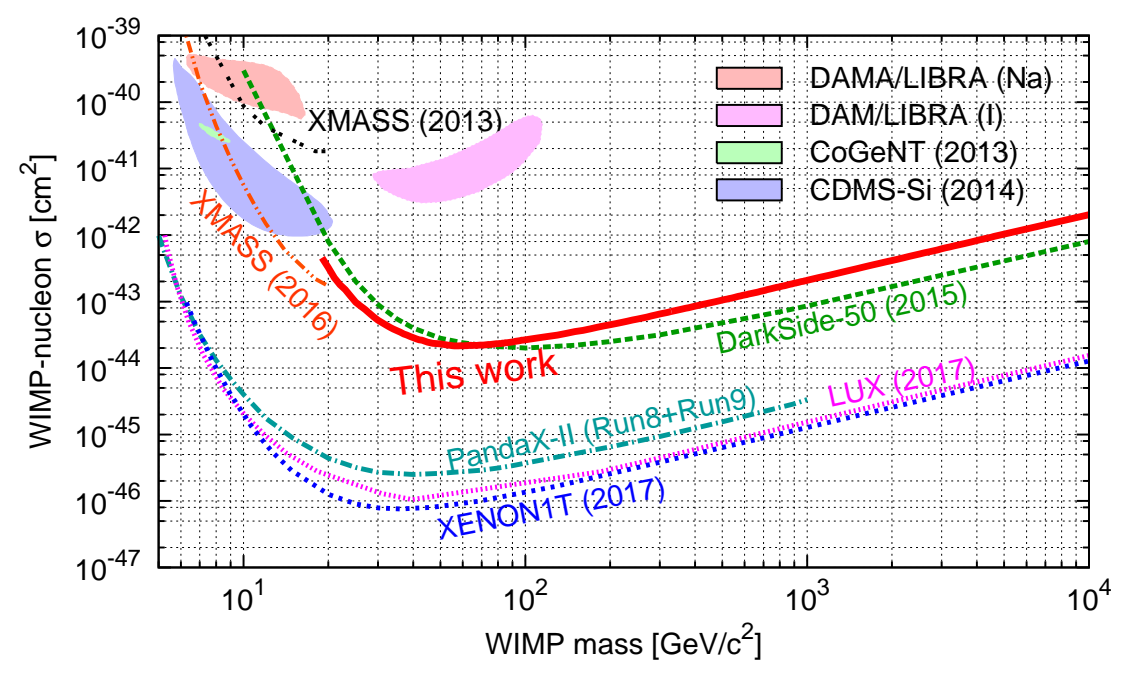

Figure 3: (Preliminary)The spin-independent WIMP-nucleon cross section limits as a function of WIMP mass at $90 \%$ confidence level for this work by solid (red in color online) line. Limits as well as allowed regions from other experimental results are shown [2, $8,10,11,12,13,14$.

[7] J.D. Lewin, P.F. Smith, Astropart. Phys. 6 (1996) 87.

[8] R. Bernabei, et al., Eur. Phys. J. C 56 (2008) 333; R. Bernabei, et al., Eur. Phys. J. C 67 (2010) 39

[9] C.E. Aalseth, et al., CoGeNT collaboration, Phys. Rev. D 88 (2013) 012002.

[10] R. Agnese, et al., CDMS collaboration, Phys. Rev. Lett. 111 (2013) 251301.

[11] P. Agnes, et al., DarkSide collaboration, Phys. Rev. D 93 (2016) 081101.

[12] A. Tan, et al., PandaX-II collaboration, Phys. Rev. Lett. 117 (2016) 121303.

[13] D.S. Akerib, et al., LUX collaboration, Phys. Rev. Lett. 118 (2017) 021303.

[14] E. Aprile, et al., XENON collaboration, arXiv:1705.06655v2. 